\title{
The Interrelationship Among Non-Alcoholic Fatty Liver Disease, Colonic Diverticulosis and Metabolic Syndrome
}

\author{
Tamara Milovanovic ${ }^{1,2}$, Ivana Pantic ${ }^{1}$, Sanja Dragasevic ${ }^{1,2}$, Sofija Lugonja $^{3}$, Igor Dumic ${ }^{4,5}$, Mirjana Rajilic-Stojanovic $^{6}$
}

1) Clinic of Gastroenterology and Hepatology, Clinical Center of Serbia, Belgrade, Serbia

2) Faculty of Medicine, University of Belgrade, Belgrade, Serbia

3) General hospital "Djordje Joanovic", Department of Internal medicine, Divison of gastroenterology, Zrenjanin, Serbia

4) Mayo Clinic Alix School of Medicine, Rochester, MN, USA

5) Mayo Clinic Health System, Eau Claire, WI, USA

6) Faculty of Technology and Metallurgy, University of Belgrade,

Belgrade, Serbia

\begin{abstract}
Non-alcoholic fatty liver disease and colonic diverticulosis are widespread, obesity-related diseases. It has recently become clear that non-alcoholic fatty liver disease is a systemic disease and may play a key role in metabolic syndrome; therefore, the term metabolic-dysfunction-associated fatty liver disease has been introduced in the literature. Excess visceral adipose tissue is an important predictor of complications in both non-alcoholic fatty liver disease and colonic diverticulosis. Current evidence suggests that intestinal dysbiosis may be involved in the development of both non-alcoholic fatty liver disease and colonic diverticulosis, and that metabolic syndrome is a consequence rather than a cause of this complex relationship. In this review, our aim was to assess the current knowledge of the complex interplay between metabolic syndrome, non-alcoholic fatty liver disease, and colonic diverticulosis.
\end{abstract}

Key words: non-alcoholic fatty liver disease - diverticulosis - metabolic syndrome - metabolic-dysfunctionassociated fatty liver disease.

Abbreviations: AD: acute diverticulitis; ADD: asymptomatic diverticular disease; BMI: body-mass index; CD: colonic diverticulosis; CDD: complicated diverticular disease; HTA: arterial hypertension; MAFLD: metabolic-dysfunction-associated fatty liver; MetS: metabolic syndrome; NAFLD: non-alcoholic fatty liver disease; NASH: non-alcoholic steatohepatitis; PCOS: polycystic ovaries syndrome; SAT: subcutaneous adipose tissue; SUDD: symptomatic uncomplicated diverticular disease; T2DM: type 2 diabetes mellitus; VAT: visceral adipose tissue.

\section{INTRODUCTION}

Metabolic syndrome Address for correspondence: Tamara Milovanovic, MD, $\mathrm{PhD}$

Clinic of Gastroenterology and Hepatology, Clinical Center of Serbia, Belgrade, Serbia; Faculty of Medicine, University of Belgrade, 11000 Belgrade, Serbia. tamara.alempijevic@med. bg.ac.rs
Received: 10.12.2020

Accepted: 15.03.2021
(MetS) is characterized by abdominal obesity, insulin resistance, hypertension, and dyslipidemia [1]. Its prevalence has increased worldwide and it can be characterized as a growing epidemic with a significant impact on healthcare costs [2]. The relationship between MetS and a broad spectrum of different conditions has been widely investigated, particularly in the context of gastrointestinal and liver disorders.

Non-alcoholic fatty liver disease (NAFLD) used to be considered a hepatic manifestation of MetS. However, new insights indicate that NAFLD is a systemic disease closely associated with MetS, and it is considered both a cause and consequence of MetS. Non-alcoholic fatty liver disease often occurs together with cardiovascular diseases, chronic kidney disease, type 2 diabetes mellitus (T2DM), polycystic ovary syndrome, psoriasis, malignancies, osteoporosis, and central obesity. Indeed, its relationship with these conditions is more complex than previously thought $[3,4]$.

Extra-hepatic manifestations of NAFLD may be linked to the chronic low-grade inflammation observed in metabolic disorders [3]. Changes in gastrointestinal microbiota and accumulation of visceral adipose tissue (VAT) (responsible for increased production of both adipocytokines and classical cytokines) cause protracted inflammation in NAFLD. Emerging evidence suggests that the interleukin-1 family of cytokines, which are associated with pro- and anti-inflammatory effects, are the essential mediators in the so-called adipose tissue-liver crosstalk [4-6].

Metabolic-dysfunction-associated fatty liver disease (MAFLD) is defined as the presence of hepatic steatosis (confirmed by liver biopsy, imaging methods, or blood 
biomarkers) and is characterized by at least one of the following three criteria: 1) being overweight or obese, 2) presence of T2DM, and 3) presence of metabolic dysregulation. The new definition of MAFLD omits the importance of alcohol consumption or other concomitant liver disease (Fig. 1) [7].

Diverticulosis is defined as the presence of diverticula and is a common anatomical condition related to aging [8]. Colonic diverticulosis (CD) presents with a wide spectrum of clinical manifestations, ranging from asymptomatic disease to segmental colitis [9-11]. Although it is advised that use of the term "diverticular disease" should be avoided, for the purpose of this review (in order to achieve uniform presentation of the available data), the following terms will be used: asymptomatic diverticular disease (ADD), symptomatic uncomplicated diverticular disease (SUDD), and complicated diverticular disease (CDD). Patients in which diverticulosis is an accidental finding are considered to have ADD. On the other hand, patients who report chronic gastrointestinal symptoms, such as changes in bowel habits, abdominal cramping, and discomfort, are diagnosed with SUDD. Complicated diverticular disease is diagnosed in the presence of more severe clinical conditions, such as acute diverticulitis (AD), diverticular bleeding, abscess and/or fistula development $[10,11]$. The role of VAT and subsequent, chronic, low-grade inflammation is important in both the pathogenesis of $\mathrm{CD}$ and its complications [12, 13], which has led many researchers to further investigate the possible complex connection of CD and MetS [14, 15]. Components of MetS, such as central obesity, dyslipidemia, and arterial hypertension (HTA), increase the risk of CDD. However, the exact impact of metabolic risk factors on the prevalence of CD has not been clearly defined [16-19].

Our aim was to synthetize the current understanding of this complex interplay between CD, NAFLD, and MetS, given the shared pathophysiological traits and scarcity of data regarding the interrelationship between these diseases.

\section{THE IMPACT OF NAFLD AND COLONIC DIVERTICULOSIS ON METABOLIC SYNDROME}

The incidence and prevalence of NAFLD is increasing, and currently, $24 \%$ of the world population is diagnosed with this disease. While almost one-third of the population in the Middle East and South America have NAFLD, its prevalence is slightly lower in Asia, USA, and Europe (27\%, 24\%, and 23\%, respectively). Notably, its incidence and prevalence are the lowest in Africa (14\%) [20]. Both NAFLD and CD are more severe in the elderly [8]. Additionally, the incidence of NAFLD

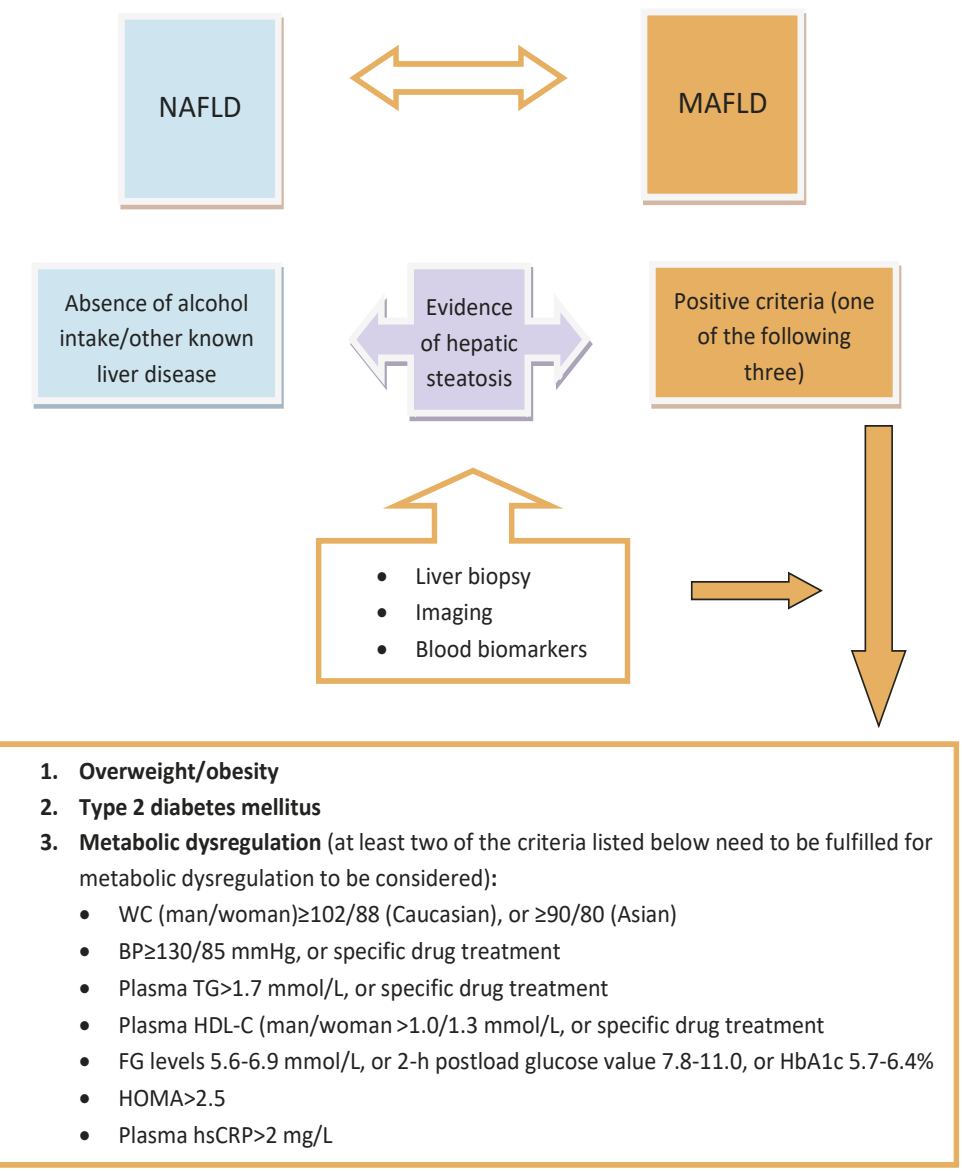

Fig. 1. Criteria for MAFLD diagnosis. BP: blood pressure; HDL-C: high-density lipoprotein; HOMA: Homeostatic Model Assessment for Insulin Resistance; hsCRP: high-sensitivity C-reactive protein; NAFLD: non-alcoholic fatty liver disease; MAFLD: metabolic-dysfunction-associated fatty liver disease; TG: triglycerides; FG: fasting glucose; WC: waist circumference. 
has an 'inverted U-shape' distribution, with a decreasing rate of newly diagnosed cases after the $6^{\text {th }}$ decade in men and the $7^{\text {th }}$ decade in women $[21,22]$. Nevertheless, NASH and cirrhosis that result from chronic inflammation typically develop in advanced age [23]. Colonic diverticulosis is also more common in Western countries, underlying the impact of Western diet, lifestyle, obesity, and MetS on development of CD $[9,18,22]$. Consequently, it appears that dietary habits and aging significantly influence the occurrence and severity of NAFLD and CD.

Recent research shows that typically CD and NAFLD occur concomitantly. In a retrospective study investigating accompanying diseases in patients with NAFLD, Kempiński et al. [24] determined that CD is the second most frequent concomitant gastrointestinal disease, second only to gastroesophageal reflux disease. Colonic diverticulosis was significantly more prevalent in the study group than in the controls (23.7\% vs. 15.8\%; p<0.005) [24]. Bae et al. [19] evaluated risk factors associated with ADD. The overall prevalence of diverticulosis was $8.1 \%$; alternatively, it was $69.7 \%$ among patients with fatty liver disease [19]. While the presence of T2DM, hyperlipidemia, smoking status, and alcohol consumption were similar in patients with $\mathrm{CD}$ and the control group, HTA was significantly more frequent in patients with ADD. This study reported that moderate and severe fatty liver disease, as well as waist-hip ratio, were risk factors for ADD, highlighting the possible role of central obesity and NAFLD in the pathogenesis of ADD [18]. Contrary to these findings, Sahin et al. [21] found that in elderly patients, diverticulosis is a negative predictor of liver steatosis. According to this study, diverticulosis could be an indirect sign of malnutrition in patients over 75 years of age, since higher values of albumin and triglycerides, were identified as independent predictors of liver steatosis [21]. The age difference between the patients included in these studies may be the reason for these conflicting findings.

\section{GUT MICROBIOTA: A POSSIBLE KEY LINK BETWEEN NAFLD AND COLONIC DIVERTICULOSIS?}

Altered gut microbiota has been identified as a possible shared factor in the pathogenesis of CD and NAFLD. Recent data have shown that specific microbial species, and the metabolites that they produce, can directly impact human metabolism [25].

It was not until recently that solid evidence emerged from controlled experimental trials that linked gut microbiome and NAFLD. Gut microbiota transplants to germ-free animal models could result in the transfer of the fatty liver disease phenotype [26]. Initial fecal microbiota transplantation from conventional mice to germ-free mice was linked to the transfer of the obesity phenotype [27] and demonstrated that transfer of gut microbiota from mice with NAFLD to germ-free mice resulted in the transfer of NAFLD features, including hyperglycemia and steatosis [26]. The same study showed that gut microbiota affects lipid metabolism in the liver, independent of obesity.

In animal models, it was shown that microbiota may be used for therapeutic purposes, since the transfer of this ecosystem from healthy, lean animals to a mouse model of NAFLD (high-fat diet-induced steatohepatitis) could induce a decrease in lipid accumulation in the liver, as well as serum transaminase levels [28]. Also, intestinal microbial dysbiosis has been reported in patients with NAFLD and non-alcoholic steatohepatitis (NASH) [29-32]. Disturbances in intestinal microbiota, accompanied by impaired gut permeability, can lead to enhanced hepatic exposure to both gut microbiota and its products, which may promote liver inflammation and subsequent fibrogenesis $[33,34]$. Microbiota can produce various metabolites, including ethanol, which may contribute to the development of NAFLD, with a similar pathophysiology as described for alcoholic fatty liver disease. Notably, children with NASH have increased abundance of alcohol-producing bacteria and elevated blood-ethanol concentration [29].

Changes in gut motility can contribute to bacterial overgrowth and nutrient malabsorption [8]. Animal studies have suggested that a diet high in cholesterol, fructose, and especially fat can cause loss of up to $30 \%$ of enteric neurons, the main regulators of gut motility $[8,35]$. However, it is unclear whether reduced gut motility contributes to the progression of NAFLD. Steatohepatitis and liver fibrosis have been associated with enteric neural loss, and morphometric analysis determined that $\mathrm{CD}$ is also associated with structural alterations of enteric neurons $[34,36]$. In addition to diet, intestinal dysbiosis has a significant impact on gut motility. We are getting closer to identifying biological mechanisms involved in gut-liver axis in animal models; however, it remains unclear whether these processes independently, or more likely, jointly, initiate the pathophysiological cascade, and eventually create a "circulus vitiosus" responsible for the development of these disorders (Fig. 2).

One of the pioneering studies by Ley et al. [37] reported that obese subjects have fewer Bacteroidetes species and a greater abundance of Firmicutes species compared to healthy controls. Some of the subsequent studies could not confirm this finding; however, the Firmicutes/Bacteroidetes ratio remained a frequently studied marker for microbiota dysbiosis in relation to diabetes and metabolic diseases [38].

Mouzaki et al. [29] reported that patients with NASH had a decreased abundance of Bacteroidetes compared to patients with simple liver steatosis and healthy subjects. A higher abundance of fecal Clostridium coccoides (corresponding to the Lachnospiraceae family) [39] was found in patients with NASH compared to those with simple steatosis. However, after correction for fat intake and BMI, this difference was not significant [29]. Yet another study confirmed this trend and verified an increase in abundance of the phylum Firmicutes, including Lactobacillus, but also several genera within the Lachnospiraceae family (Dorea, Roseburia, and Robinsoniella) in patients with NAFLD compared to healthy subjects [31]. In addition, Loomba et al. [32] assessed gut microbiome compositions in patients with mild or moderate NAFLD and advanced fibrosis using whole-genome shotgun sequencing of DNA extracted from stool samples. They found a higher prevalence of Firmicutes in those with mild or moderate NAFLD, while Proteobacteria were the dominant microbiota in those with liver fibrosis [32]. Wang B et al. [30] investigated intestinal dysbiosis in non-obese patients with NAFLD. They 


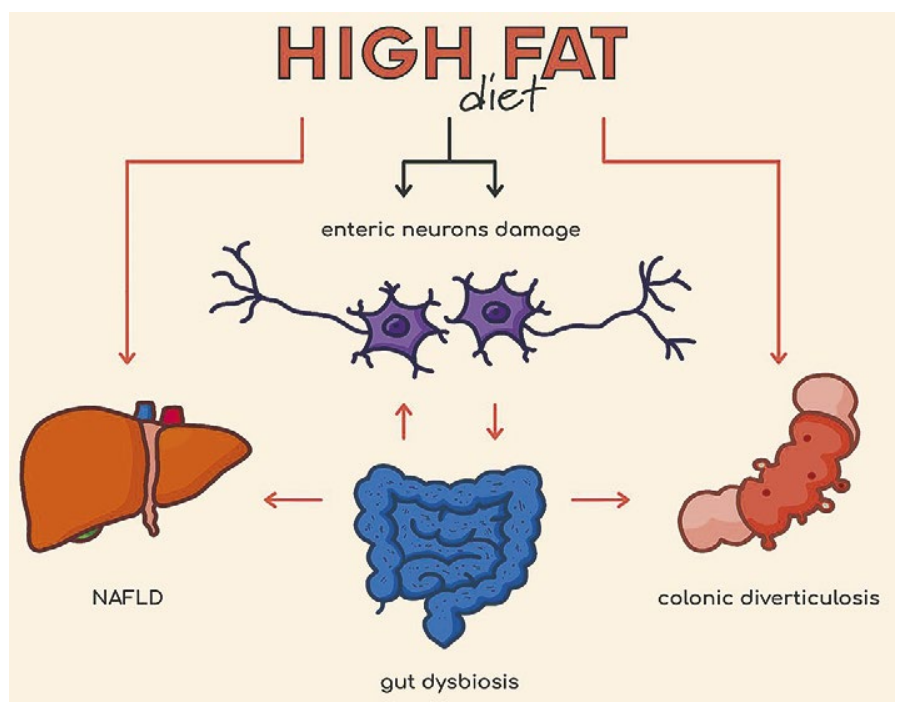

Fig. 2. Overlapping pathophysiological cascade of NAFLD and CD. NAFLD: non-alcoholic fatty liver disease.

reported opposite trends for the Bacteroidetes phylum, which was detected in up to $20 \%$ of patients with NALFD compared to lean individuals. In obese adults, the Firmicutes phylum, particularly the Lachnospiraceae family, were reduced in abundance compared to healthy, non-obese adults [30]. This was also true for the Lactobacillaceae family, and for bile acid dehydroxylating Ruminococcaceae. All these studies have linked impaired gut microbiota not only with the development of NAFLD, but also its progression and disease severity.

Profiling studies of the microbiota in CD have highlighted that the overgrowth of the phylum Firmicutes (Ruminococcus, Pseudobutyrivibrio, and Christensenellaceae family) may be associated not only with SUDD, but also with AD occurrence [40, 41]. Indeed, diverticular pockets may represent unique niches that selectively promote the development of specific microbes, leading to inflammation and clinical manifestations of CD.

Barbara et al. [42] and Tursi et al. [43] investigated the difference in fecal microbiota between patients with uncomplicated CD and healthy individuals. They verified reduced abundance of Akkermansia muciniphila in the diverticular region compared with sites in the distal colon. They also observed a negative correlation between macrophage counts in CD and Akkermansia abundance [42]. Notably, patients with CD had significantly higher macrophage counts, which was negatively correlated with the abundance of the Clostridium cluster IV, which includes anti-inflammatory Faecalibacterium Prausnitzii. Therefore, it has been proposed that patients with CD lack anti-inflammatory microbiota species [42]. Indeed, a difference was noted in the microbiota of patients with SUDD and patients with ADD. Patients with SUDD had significantly reduced abundance of the phylum Firmicutes (Clostridium cluster IX, Lactobacillaceae) and Fusobacterium compared to asymptomatic individuals [43]. These findings are surprising, since increased abundance of Fusobacterium is a strong marker of various pathologies, including appendicitis [44] and colon cancer [45].

Patterns of microbiota dysbiosis assessed through metabolomics and gut microbiota diversity showed several pro-inflammatory (microbial) metabolites in patients with
CD compared to controls. Barbara et al. [42] suggested that metabolome profiles associated with inflammatory pathways and bowel neuromotor disorders may distinguish patients with $\mathrm{CD}$ and healthy subjects. The results of the study by Tursi et al. [43] are not in line with the findings of Barbara et al. [43], as they only noted differences in fecal abundance of Akkermasia muciniphila in controls compared to patients with ADD and patients with SUDD.

In their attempt to highlight the significance of microbial metabolic output in relation to the pathogenesis of $\mathrm{CD}$, Barbara et al. [42] showed that a combination of microbiological and metabolic profiling allows for separation between $\mathrm{ADD}$ and controls, mainly due to the change in Akkermasia muciniphila abundance. Indeed, Akkermasia muciniphila abundance is negatively correlated with levels of N-acetylglucosamine. Interestingly, microbiological and metabolic data allowed for separation between ADD and SUDD based on $\mathrm{N}$-acetyl-glucosamine levels and several short-chain fatty acids [43]. In another study, profiles of microbiota were examined and compared between patients with SUDD who were previously diagnosed with $\mathrm{AD}$ and those who were not [41]. It was established that recurrent $\mathrm{AD}$ could be associated with a specific microbiota signature marked by the increased prevalence of Pseudobutyrivibrio and Bifidobacterium spp. and decreased prevalence of uncultured species belonging to the Christensenellaceae family and the Mollicutes order [41]. In this study, a correlation between symptoms, such as bloating and pain, and particular members of the gut microbiota was identified. The limitations of current studies are the small sample size, discrepancy between applied techniques, and scarce clinical assessment of CD, resulting in conflicting and/ or inconsistent conclusions. Specifically, it seemed that the role of microbiota in the early phases of ADD is overlooked. Nevertheless, there was ample evidence that significant microbiota alterations are associated with complications of CD.

Since gut microbiota composition in NAFLD has also been linked to disease severity and progression [24], the aforementioned findings could indicate the potential for shared traits in the progression of both CD and NAFLD. 


\section{OBESITY IN NAFLD AND COLONIC DIVERTICULOSIS}

Visceral adiposity, with associated pro-inflammatory effects, is a major risk factor for NAFLD and development of symptomatic CD $[33,46]$. Several studies have reported a prevalence of NAFLD up to $90 \%$ in patients undergoing bariatric surgery for morbid obesity. Although simple hepatic steatosis of various degrees was the most common clinical finding, NASH was diagnosed in up to $50 \%$ of these patients [47-49].

Kopylov et al. [19] demonstrated an association between obesity and CD; alternatively, Song et al. [50] determined that typically patients with CD have higher BMI. Furthermore, obesity expressed not only as BMI, but also as waist circumference and waist-to-hip ratio, has recently been highlighted as one of the strongest risk factors for CDD [12, 15]. In a Swedish cohort study with over 28 -years of followup, which included subjects hospitalized for CDD, BMI was found to be a significant risk factor for CD [15]. Strate et al. [12] studied male health professionals with self-reported diverticulitis and diverticular disease (confirmed by assessment of the medical records) and reported that subjects with BMI $>30$ $\mathrm{kg} / \mathrm{m}^{2}$ had a relative risk of 1.78 (95\% confidence interval (CI), 1.08-2.94) of developing diverticulitis, and 3.19 (95\% CI, 1.45-7.00) of diverticular bleeding, compared to non-obese individuals.

Alfonso et al. [51] assessed both visceral and subcutaneous adipose tissue (SAT) via ultrasound, and further evaluated the association between SAT and CD. They reported that in addition to older age, VAT is an independent risk factor for CD. A few studies that used computed tomography also highlighted VAT, and not BMI, as an independent risk factor for left-sided CD $[52,53]$. The significance of VAT as a risk factor for leftsided diverticulosis in Japan was reported in a study by Yamada et al. [54], indicating that patients with left-sided diverticulitis are more likely to develop diverticulitis when the VAT area is greater than $100 \mathrm{~cm}^{2}$.

Similarly, Kim et al. [55] conducted a longitudinal study in adults who underwent abdominal ultrasonography, with a 4.4-year follow-up period, and suggested that larger areas of VAT at baseline are linked to higher NAFLD occurrence, with a hazard ratio of 2.23; additionally, an association with SAT was not identified. Interestingly, regression of NAFLD during the follow-up was noticed in subjects who had significantly higher baseline SAT areas, irrespective of the VAT area. Therefore, the authors proposed a possible beneficial effect of SAT on the disease course of NAFLD [55]. Additionally, the authors reported that an increase in VAT over time correlates with a higher probability of NAFLD development, independent of the baseline VAT area [56]. Conversely, a decrease in the VAT area had a protective effect on the disease course of NAFLD and led to disease regression [57]. Similar findings were reported by other authors who demonstrated that the VAT area is associated with both NASH and fibrosis development, with higher areas of VAT being associated with more severe disease [57].

Visceral adipose tissue is an important predictor of metabolic and intestinal complications in obesity-related disorders, including NAFLD and CD. The complex endocrine role of VAT includes the release of various hormones, including adiponectin and leptin. While leptin has pro-inflammatory activity, adiponectin acts as an anti-inflammatory agent [33, 46]. The role of adiponectin and leptin in obesity, insulin resistance, and NAFLD has been shown in several studies using human, animal, and in vitro models. However, studies addressing the specific role of these hormones in CD are scarce $[33,46,58]$. Tsochatzis et al. [59] suggested that both a reduction in adiponectin levels and increased leptin in obese individuals contribute to the development of hepatic steatosis, increased inflammation, and fibrogenesis of the liver tissue. Murray et al. [45] reported that adiponectin levels showed a negative correlation with VAT volume in patients with CD.

The exact role of abdominal obesity in the pathogenesis of $\mathrm{CD}$ remains unclear. The herniation of colonic mucosa due to an obesity-related increase in intraluminal pressure is also aggravated by methane gas production resulting from intestinal dysbiosis observed in obese individuals. Additionally, adipocytes produce pro-inflammatory cytokines that can influence colonic motility, which promotes diverticula formation $[9,20]$. Studies investigating the specific role of obesity and metabolic factors in $\mathrm{CD}$ have shown varying results $[14,19,53]$. In a Southern-European prospective study, a positive association between increased waist circumference, blood pressure, hyperlipidemia, and CD was reported; also, there was no significant difference in the prevalence of T2DM and obesity between those who had CD and those who did not. Furthermore, multivariate analysis revealed that advanced age and greater waist circumference constitute independent risk factors for CD [14]. In a five-year longitudinal study by Kopylov et al. [19], the following factors were associated with increased risk of $\mathrm{CD}$ : age, male gender, BMI, obesity, systolic blood pressure, low-density lipoprotein cholesterol level, history of hypertension, ischemic heart disease, hypothyroidism, and absence of T2DM.

\section{ARTERIAL HYPERTENSION AS A RISK FACTOR IN NAFLD AND COLONIC DIVERTICULOSIS}

Almost half of the patients with HTA develop NAFLD. Several studies have indicated a strong association between increased blood pressure and NAFLD in both hypertensive and normotensive patients [60-62]. However, most of these studies were cross-sectional; therefore, it is not possible to determine the causal relationship between NAFLD and HTA.

The study by Ma et al. [63] highlighted the mutual relationship between NAFLD and HTA, emphasizing its bi-directional nature, and suggested that NAFLD could be both a cause and consequence of HTA. Several studies have addressed the interaction between elevated blood pressure and the development of $\mathrm{CD}$. One of the first studies regarding this association, conducted by Yeo et al. [64], concluded that patients with HTA had a higher probability of developing ADD. This study further emphasized that inadequately regulated HTA is a significant risk factor for the development of ADD. A positive correlation between HTA and CD has been previously reported $[14,17]$. Indeed, the atherogenic potential of HTA is well known. Considering that diverticula most commonly 
occur in vulnerable spots of the colonic wall (the site where vasa recta passes through the circular muscular layer), it is possible to conclude that HTA, especially when inadequately regulated, may lead to structural changes in the bowel wall over time by damaging blood vessels and further reducing blood supply in vulnerable anatomical areas. Interestingly, the use of calcium channel blockers for the treatment of HTA appears to have a protective effect against CD [8].

\section{THE IMPACT OF DIABETES MELLITUS TYPE 2 ON NAFLD AND COLONIC DIVERTICULOSIS}

The reciprocity of NAFLD and metabolic disorders, including T2DM, has recently become an area of interest for both clinicians and researches. Changes in liver structure in NAFLD can contribute to the development of T2DM in predisposed individuals via hepatic insulin resistance. Hepatic ceramides and diacylglycerols are identified as the most important mediators of insulin resistance and play a major role in induction of lipotoxicity in numerous metabolic disorders. It has been suggested that the inhibition of their synthesis could lead to improvement of NAFLD, and consequently, insulin resistance, thereby preventing insufficiency of pancreatic beta cells [65]. Insulin resistance, as a central mechanism for the development of both NAFLD and T2DM, could be mediated by the production of a microbial metabolite, imidazole-propionate [66]. Imidazole-propionate is produced from the amino acid histidine by specific components of the microbiota, and this microbial metabolite can impair insulin signaling at the level of the insulin receptor (through activation of p38 $\gamma$ MAPK) [66]. Interestingly, the drug most prescribed in insulin resistance treatment, metformin, has been shown to influence colonic microbiota composition [67]. A recent study demonstrated that imidazole-propionate inhibits metformin activity through imidazole-propionate-activated p38 $\gamma$ [68]. Metformin has recently been used in various animal and human studies, and it reportedly has an anti-inflammatory role in CD $[69,70]$.

Type 2 diabetes mellitus, NAFLD, and CD are linked in a number of ways. Several studies have indicated that a low-fiber diet is associated with both T2DM [71] and cardiovascular disease development [72,73]. As previously stated, the following factors are associated with an increased risk for $\mathrm{CD}$ : advanced age, male gender, obesity, history of hypothyroidism, and absence of T2DM [19]. However, T2DM may play a role in the occurrence of diverticular bleeding [74]. Longlasting T2DM is associated with numerous complications, including disruption of mucosal integrity, which may also be considered as a contributing factor to diverticular bleeding. A recently conducted meta-analysis assessed cohort studies on the relationship between $\mathrm{CD}$ and the following factors: age, gender, BMI, HTA, smoking, finger prick test glucose levels, $\mathrm{HbA1c}$ levels, and C-reactive protein levels [75]. It concluded that diabetes is an important risk factor for both CD and the development of diverticular bleeding. Patients with diabetes had approximately a 1.25 -fold higher risk for diverticulosis, and approximately a 2.3-fold higher risk of colectomy, which was the only therapeutic option for diverticular bleeding. The main limitation of this meta-analysis is that only several prospective and randomized cohort studies were included, and that both patients with type 1 and type 2 diabetes mellitus were jointly analyzed [75]. Therefore, it may be possible to decrease the risk of recurrent and potentially life-threatening diverticular bleeding by providing the patient with adequate glucose regulation.

\section{CONCLUSIONS}

This review highlights the complex interrelationship and shared traits between NAFLD, CD, and MetS (Fig. 3, Table I). In the past, MetS was believed to be the cause of several clinical conditions traditionally associated with metabolic abnormalities.

Table I. Summary of CD and NAFLD meeting points

\begin{tabular}{|c|c|c|c|c|}
\hline Reference & Colonic diverticulosis & $\begin{array}{l}\text { Meeting } \\
\text { points }\end{array}$ & Non-alcoholic fatty liver disease & Reference \\
\hline 41 & $\begin{array}{l}\text { Deficiency of anti-inflammatory gut } \\
\text { microbiota components }\end{array}$ & Dysbiosis & $\begin{array}{l}\text { Impaired gut microbiota linked with hepatic fat deposition, } \\
\text { progression of NAFLD and with the disease severity }\end{array}$ & $\begin{array}{l}25,26,27 \\
28,29,30,31\end{array}$ \\
\hline 39,40 & $\begin{array}{l}\text { Correlation between gut microbiota } \\
\text { signature and the disease severity }\end{array}$ & & Pro-inflammatory effect & 32,33 \\
\hline $\begin{array}{l}11,13,14 \\
45,50,53\end{array}$ & $\begin{array}{l}\text { Risk factor for CD development and } \\
\text { its complications }\end{array}$ & $\begin{array}{l}\text { Visceral } \\
\text { adiposity }\end{array}$ & $\begin{array}{l}\text { Risk factor for NAFLD development and more advanced } \\
\text { disease course }\end{array}$ & $32,54,55,56$ \\
\hline 18,49 & $\begin{array}{l}\text { High prevalence of CD in obese } \\
\text { subjects }\end{array}$ & & & \\
\hline 13,16 & $\begin{array}{l}\text { Positive correlation between HTA } \\
\text { and CD }\end{array}$ & $\begin{array}{l}\text { Arterial } \\
\text { hypertension }\end{array}$ & $\begin{array}{l}\text { NAFLD is associated with an independent risk of identifying } \\
\text { HTA }\end{array}$ & 59,62 \\
\hline \multirow[t]{2}{*}{63} & $\begin{array}{l}\text { HTA is increasing the risk of } \\
\text { asymptomatic CD development }\end{array}$ & & HTA is an independent risk factor for NAFLD development & $60,62,63$ \\
\hline & & & HTA correlates with liver fat content & 61 \\
\hline \multirow[t]{2}{*}{74} & $\begin{array}{l}\text { T2DM increases the risk of } \\
\text { complicated CD }\end{array}$ & $\begin{array}{l}\text { Type } 2 \\
\text { diabetes } \\
\text { mellitus }\end{array}$ & $\begin{array}{l}\text { Insulin resistance as a pathogenic mechanism described in } \\
\text { both NAFLD and T2DM }\end{array}$ & 64 \\
\hline & & & Insulin resistance is related to higher incidence of fatty liver & 60,62 \\
\hline
\end{tabular}

CD: colonic diverticulosis; HTA: arterial hypertension; NAFLD: nonalcoholic liver disease; T2DM: type 2 diabetes mellitus. 


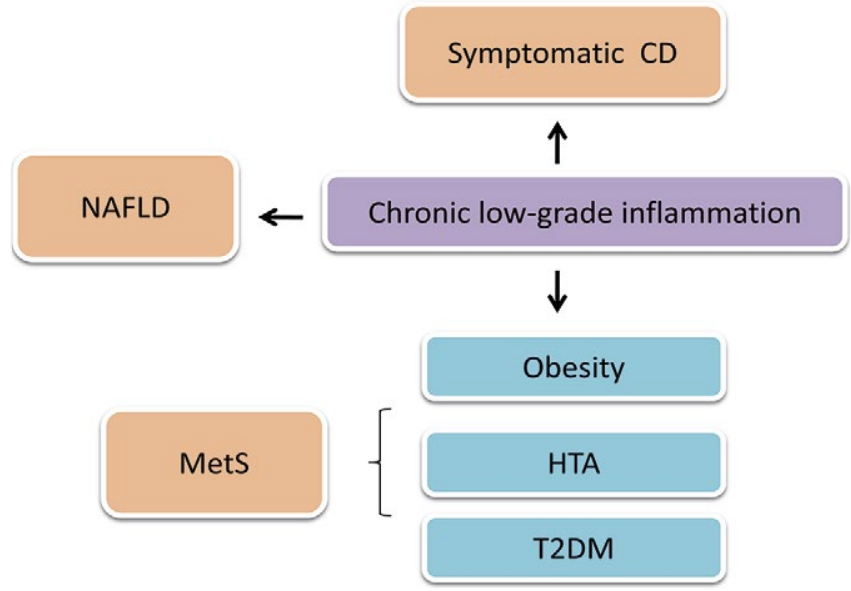

Fig. 3. The relationship between NAFLD, MetS and CD. CD: colonic diverticulosis; NAFLD: non-alcoholic fatty liver disease; MetS: metabolic syndrome; HTA: arterial hypertension; T2DM: type 2 diabetes mellitus.

Herein, we provide a different perspective by analyzing evidence of the reverse sequence of pathophysiological events by considering disturbances in gut microbiota as the cause of NAFLD and CD (especially symptomatic forms of the disease). Alternatively, MetS is considered a consequence of NAFLD and $\mathrm{CD}$, rather than the cause of these conditions. Currently, it remains difficult to establish a strict pathophysiological timeline and define the underlying causative mechanisms of NAFLD. Nevertheless, the bi-directional relationship between these three diseases (NAFLD, CD, and MetS) implies that further studies are needed to develop novel therapeutic approaches and efficient treatment strategies.

Conflicts of interest: None to declare.

Authors' contributions: T.M., I.P., S.D., S.L., I.D., M.R.S. conceived the study and searched the relevant literature. I.P., S.D., S.L., I.D., M.R.S. drafted the manuscript. T.M., I.P., S.D., S.L., I.D., M.R.S. revised the paper. T.M supervised the study. All authors critically revised the manuscript, approved the final version to be published, and agree to be accountable for all aspects of the work.

\section{REFERENCES}

1. Mendrick DL, Diehl AM, Topor LS, et al. Metabolic Syndrome and Associated Diseases: From the Bench to the Clinic. Toxicol Sci 2018;162:36-42. doi:10.1093/toxsci/kfx233

2. Saklayen MG. The Global Epidemic of the Metabolic Syndrome. Curr Hypertens Rep 2018;20:12. doi:10.1007/s11906-018-0812-Z

3. Li AA, Ahmed A, Kim D. Extrahepatic manifestations of nonalcoholic fatty liver disease. Gut Liver 2020;14:168-178. doi:10.5009/gnl19069

4. Adams LA, Anstee QM, Tilg H, Targher G. Non-Alcoholic fatty liver disease and its relationship with cardiovascular disease and other extrahepatic diseases. Gut 2017;66:1138-1153. doi:10.1136/ gutjnl-2017-313884

5. Tilg H, Moschen AR, Szabo G. Interleukin-1 and inflammasomes in alcoholic liver disease/acute alcoholic hepatitis and nonalcoholic fatty liver disease/nonalcoholic steatohepatitis. Hepatology 2016;64:955-965. doi:10.1002/hep.28456
6. Pihlajamäki J, Kuulasmaa T, Kaminska D, et al. Serum interleukin 1 receptor antagonist as an independent marker of non-alcoholic steatohepatitis in humans. J Hepatol 2012;56:663-670. doi:10.1016/j. jhep.2011.10.005

7. Eslam M, Newsome PN, Sarin SK, et al. A new definition for metabolic dysfunction-associated fatty liver disease: An international expert consensus statement. J Hepatol 2020;73:202-209. doi:10.1016/j. jhep.2020.03.039

8. Dumic I, Nordin T, Jecmenica M, Stojkovic Lalosevic M, Milosavljevic T, Milovanovic T. Gastrointestinal Tract Disorders in Older Age. Can J Gastroenterol Hepatol 2019;2019:6757524. doi:10.1155/2019/6757524

9. Tursi A, Scarpignato C, Strate LL, et al. Colonic diverticular disease. Nat Rev Dis Primers 2020;6:20. doi:10.1038/s41572-020-0153-5

10. Scarpignato C, Barbara G, Lanas A, Strate LL. Management of colonic diverticular disease in the third millennium: Highlights from a symposium held during the United European Gastroenterology Week 2017. Therap Adv Gastroenterol 2018;11:1756284818771305. doi:10.1177/1756284818771305

11. Klarenbeek BR, De Korte N, Van Der Peet DL, Cuesta MA. Review of current classifications for diverticular disease and a translation into clinical practice. Int J Colorectal Dis 2012;27:207-214. doi:10.1007/ s00384-011-1314-5

12. Strate LL, Liu YL, Aldoori WH, Syngal S, Giovannucci EL. Obesity increases the risks of diverticulitis and diverticular bleeding. Gastroenterology 2009;136:115-122.e1. doi:10.1053/j. gastro.2008.09.025

13. Beasley LE, Koster A, Newman AB, et al. Inflammation and race and gender differences in computerized tomography-measured adipose depots. Obesity 2009;17:1062-1069. doi:10.1038/oby.2008.627

14. Teixeira C, Trabulo D, Ribeiro S, et al. Colonic diverticulosis and the metabolic syndrome: an association? Rev Esp Enfermedades Dig 2017;109:768-771. doi:10.17235/reed.2017.5009/2017

15. Rosemar A, Angerås U, Rosengren A. Body mass index and diverticular disease: a 28-year follow-up study in men. Dis Colon Rectum 2008;51:450-455. doi:10.1007/s10350-007-9172-5

16. Freckelton J, Holt D, Borsaru A, Gwini SM, Croagh D, Moore G. The role of body composition in diverticular disease. Int J Colorectal Dis 2018;33:1299-1302. doi:10.1007/s00384-018-3058-y

17. Tursi A, Brandimarte G, Di Mario F, et al. International consensus on diverticulosis and diverticular disease. Statements from the $3 \mathrm{rd}$ international symposium on diverticular disease. J Gastrointest Liver Dis 2019;28(Suppl 4):57-66. doi:10.15403/jgld-562

18. Bae HJ, Kim ST, Hong SG, et al. Risk Factors for Asymptomatic Colon Diverticulosis. Korean J Gastroenterol 2019;74:142-148. doi:10.4166/ kjg.2019.74.3.142

19. Kopylov U, Ben-Horin S, Lahat A, Segev S, Avidan B, Carter D. Obesity, metabolic syndrome and the risk of development of colonic diverticulosis. Digestion 2012;86:201-205. doi:10.1159/000339881

20. Younossi ZM, Koenig AB, Abdelatif D, Fazel Y, Henry L, Wymer M. Global epidemiology of nonalcoholic fatty liver disease-Meta-analytic assessment of prevalence, incidence, and outcomes. Hepatology 2016;64:73-84. doi:10.1002/hep.28431

21. Sahin A, Tunc N, Demirel U, Kursat Poyrazoglu O, Yalniz M, Halil Bahcecioglu I. Relationship between diverticulosis and nonalcoholic fatty liver disease in elderly patients. J Int Med Res 2018;46:1545-1554. doi:10.1177/0300060517751740

22. Bertolotti M, Lonardo A, Mussi C, et al. Nonalcoholic fatty liver disease and aging: epidemiology to management. World J Gastroenterol 2014;20:14185-14204. doi:10.3748/wjg.v20.i39.14185 
23. Martel J, Raskin JB; NDSG. History, incidence, and epidemiology of diverticulosis. J Clin Gastroenterol 2008;42:1125-1127. doi:10.1097/ MCG.0b013e3181865f18

24. Kempiński R, Łukawska A, Krzyżanowski F, Ślósarz D, Poniewierka E. Clinical outcomes of non-alcoholic fatty liver disease: Polish-case control study. Adv Clin Exp Med 2019;28:1615-1620. doi:10.17219/acem/106173

25. Koh A, Bäckhed F. From Association to Causality: the Role of the Gut Microbiota and Its Functional Products on Host Metabolism. Mol Cell 2020;78:584-596. doi:10.1016/j.molcel.2020.03.005

26. Le Roy $\mathrm{T}$, Llopis $\mathrm{M}$, Lepage $\mathrm{P}$, et al. Intestinal microbiota determines development of non-alcoholic fatty liver disease in mice. Gut 2013;62:1787-1794. doi:10.1136/gutjnl-2012-303816

27. Bäckhed F, Ding $H$, Wang $T$, et al. The gut microbiota as an environmental factor that regulates fat storage. Proc Natl Acad Sci U S A 2004;101:15718-21573. doi:10.1073/pnas.0407076101

28. Zhou D, Pan Q, Shen F, et al. Total fecal microbiota transplantation alleviates high-fat diet-induced steatohepatitis in mice via beneficial regulation of gut microbiota. Sci Rep 2017;7:1529. doi:10.1038/s41598017-01751-y

29. Mouzaki M, Comelli EM, Arendt BM, et al. Intestinal microbiota in patients with nonalcoholic fatty liver disease. Hepatology 2013;58:120 127. doi:10.1002/hep.26319

30. Wang B, Jiang X, Cao M, et al. Altered fecal microbiota correlates with liver biochemistry in nonobese patients with non-alcoholic fatty liver disease. Sci Rep 2016;6:32002. doi:10.1038/srep32002

31. Raman M, Ahmed I, Gillevet PM, et al. Fecal microbiome and volatile organic compound metabolome in obese humans with nonalcoholic fatty liver disease. Clin Gastroenterol Hepatol 2013;11:868-875.e1-e3. doi:10.1016/j.cgh.2013.02.015

32. Loomba R, Seguritan V, Li W, et al. Gut Microbiome-Based Metagenomic Signature for Non-invasive Detection of Advanced Fibrosis in Human Nonalcoholic Fatty Liver Disease. Cell Metab 2017;25:1054-1062.e5. doi:10.1016/j.cmet.2017.04.001

33. Zhang X, Ji X, Wang Q, Li JZ. New insight into inter-organ crosstalk contributing to the pathogenesis of non-alcoholic fatty liver disease (NAFLD). Protein Cell 2018;9:164-177. doi:10.1007/s13238-017-0436-0

34. Leung C, Rivera L, Furness JB, Angus PW. The role of the gut microbiota in NAFLD. Nat Rev Gastroenterol Hepatol 2016;13:412-425. doi:10.1038/nrgastro.2016.85

35. Rivera LR, Leung C, Pustovit R V, et al. Damage to enteric neurons occurs in mice that develop fatty liver disease but not diabetes in response to a high-fat diet. Neurogastroenterol Motil 2014;26:11881199. doi:10.1111/nmo.12385

36. Wedel T, Büsing V, Heinrichs G, et al. Diverticular disease is associated with an enteric neuropathy as revealed by morphometric analysis. Neurogastroenterol Motil 2010;22:407-414.e94. doi:10.1111/j.13652982.2009.01445.x

37. Ley RE, Turnbaugh PJ, Klein S, Gordon JI. Microbial ecology: human gut microbes associated with obesity. Nature 2006;444:1022-1023. doi:10.1038/4441022a

38. Magne F, Gotteland M, Gauthier L, et al. The firmicutes/bacteroidetes ratio: a relevant marker of gut dysbiosis in obese patients? Nutrients 2020;12:1474. doi:10.3390/nu12051474

39. Rajilić-Stojanović M, de Vos WM. The first 1000 cultured species of the human gastrointestinal microbiota. FEMS Microbiol Rev 2014;38:9961047. doi:10.1111/1574-6976.12075

40. Ticinesi A, Nouvenne A, Corrente V, Tana C, Di Mario F, Meschi T. Diverticular Disease: a Gut Microbiota Perspective. J Gastrointestin Liver Dis 2019;28:327-337. doi:10.15403/jgld-277
41. Kvasnovsky CL, Leong LEX, Choo JM, et al. Clinical and symptom scores are significantly correlated with fecal microbiota features in patients with symptomatic uncomplicated diverticular disease: a pilot study. Eur J Gastroenterol Hepatol 2018;30:107-112. doi:10.1097/ MEG.0000000000000995

42. Barbara G, Scaioli E, Barbaro MR, et al. Gut microbiota, metabolome and immune signatures in patients with uncomplicated diverticular disease. Gut 2017;66:1252-1261. doi:10.1136/gutjnl-2016-312377

43. Tursi A, Mastromarino P, Capobianco D, et al. Assessment of fecal microbiota and fecal metabolome in symptomatic uncomplicated diverticular disease of the colon. J Clin Gastroenterol 2016;50Suppl 1:S9-S12. doi:10.1097/MCG.0000000000000626

44. Swidsinski A, Dörffel Y, Loening-Baucke V, et al. Acute appendicitis is characterised by local invasion with Fusobacterium nucleatum/ necrophorum. Gut 2011;60:34-40. doi:10.1136/gut.2009.191320

45. Kostic AD, Chun E, Robertson L, et al. Fusobacterium nucleatum potentiates intestinal tumorigenesis and modulates the tumor-immune microenvironment. Cell Host Microbe 2013;14:207-215. doi:10.1016/j. chom.2013.07.007

46. Murray KA, Hoad CL, Garratt J, et al. A pilot study of visceral fat and its association with adipokines, stool calprotectin and symptoms in patients with diverticulosis. PLoS One 2019;14:e0216528. doi:10.1371/ journal.pone.0216528

47. Frantzides CT, Carlson MA, Moore RE, et al. Effect of body mass index on nonalcoholic fatty liver disease in patients undergoing minimally invasive bariatric surgery. J Gastrointest Surg 2004;8:849-855. doi:10.1016/j.gassur.2004.07.001

48. Abrams GA, Kunde SS, Lazenby AJ, Clements RH. Portal fibrosis and hepatic steatosis in morbidly obese subjects: A spectrum of nonalcoholic fatty liver disease. Hepatology 2004;40:475-483. doi:10.1002/hep.20323

49. Dallal RM, Mattar SG, Lord JL, et al. Results of laparoscopic gastric bypass in patients with cirrhosis. Obes Surg 2004;14:47-53. doi:10.1381/096089204772787284

50. Song JH, Kim YS, Lee JH, et al. Clinical characteristics of colonic diverticulosis in Korea: a prospective study. Korean J Intern Med 2010;25:140-146. doi:10.3904/kjim.2010.25.2.140

51. Afonso M, Pinto J, Veloso R, Freitas T, Carvalho J, Fraga, J. Visceral fat, a key factor in diverticular disease of the colon. GE J Port Gastrenterol 2012;19:6265.

52. Lee SP, Ahn YW, Lee OY, Lee KN. The relationship between colonic diverticulosis and abdominal visceral and subcutaneous fat accumulation measured by abdominal CT scan. Turk J Gastroenterol 2014;25:192-197. doi:10.5152/tjg.2014.4581

53. Nagata N, Sakamoto K, Arai T, et al. Visceral abdominal obesity measured by computed tomography is associated with increased risk of colonic diverticulosis. J Clin Gastroenterol 2015;49:816-822. doi:10.1097/MCG.0000000000000267

54. Yamada E, Ohkubo H, Higurashi T, et al. Visceral obesity as a risk factor for left-sided diverticulitis in Japan: A multicenter retrospective study. Gut Liver 2013;7:532-538. doi:10.5009/gnl.2013.7.5.532

55. Kim D, Chung GE, Kwak MS, et al. Body Fat Distribution and Risk of Incident and Regressed Nonalcoholic Fatty Liver Disease. Clin Gastroenterol Hepatol 2016;14:132-138.e4. doi:10.1016/j. cgh.2015.07.024

56. Kim D, Chung GE, Kwak MS, Kim YJ, Yoon JH. Effect of longitudinal changes of body fat on the incidence and regression of nonalcoholic fatty liver disease. Dig Liver Dis 2018;50:389-395. doi:10.1016/j. dld.2017.12.014 
57. Yu SJ, Kim W, Kim D, et al. Visceral obesity predicts significant fibrosis in patients with nonalcoholic fatty liver disease. Medicine (Baltimore) 2015;94:e2159. doi:10.1097/MD.0000000000002159

58. Matsuzawa Y. Adiponectin: a key player in obesity related disorders Curr Pharm Des 2010;16:1896-1901. doi:10.2174/138161210791208893

59. Tsochatzis E, Papatheodoridis GV, Archimandritis AJ. The evolving role of leptin and adiponectin in chronic liver diseases. Am J Gastroenterol 2006;101:2629-2640.

60. López-Suárez A, Guerrero JMR, Elvira-González J, Beltrán-Robles M, Cañas-Hormigo F, Bascuñana-Quirell A. Nonalcoholic fatty liver disease is associated with blood pressure in hypertensive and nonhypertensive individuals from the general population with normal levels of alanine aminotransferase. Eur J Gastroenterol Hepatol 2011;23:1011-1017. doi:10.1097/MEG.0b013e32834b8d52

61. Qian LY, Tu JF, Ding YH, et al. Association of blood pressure level with nonalcoholic fatty liver disease in nonhypertensive population Normal is not the new normal. Medicine (Baltimore) 2016;95:e4293. doi:10.1097/MD.0000000000004293

62. Lorbeer R, Bayerl C, Auweter S, et al. Association between MRI-derived hepatic fat fraction and blood pressure in participants without history of cardiovascular disease. J Hypertens 2017;35:737-744. doi:10.1097/ HJH.0000000000001245

63. Ma J, Hwang SJ, Pedley A, et al. Bi-directional analysis between fatty liver and cardiovascular disease risk factors. J Hepatol 2017;66:390-397. doi:10.1016/j.jhep.2016.09.022

64. Yeo LX, Tseng TH, Chen WL, et al. Hypertension control and risk of colonic diverticulosis. Therap Adv Gastroenterol 2019;12:1756284819855734. doi:10.1177/1756284819855734

65. Kumashiro N, Erion DM, Zhang D, et al. Cellular mechanism of insulin resistance in nonalcoholic fatty liver disease. Proc Natl Acad Sci U S A 2011;108:16381-16385. doi:10.1073/pnas.1113359108

66. Koh A, Molinaro A, Ståhlman M, et al. Microbially Produced Imidazole Propionate Impairs Insulin Signaling through mTORC1. Cell 2018;175:947-961.e17. doi:10.1016/j.cell.2018.09.055
67. Shin NR, Lee JC, Lee HY, et al. An increase in the Akkermansia spp. population induced by metformin treatment improves glucose homeostasis in diet-induced obese mice. Gut 2014;63:727-735. doi:10.1136/gutjnl-2012-303839

68. Koh A, Mannerås-Holm L, Yunn NO, et al. Microbial Imidazole Propionate Affects Responses to Metformin through p38 $\gamma$-Dependent Inhibitory AMPK Phosphorylation. Cell Metab 2020;32:643-653.e4. doi:10.1016/j.cmet.2020.07.012

69. Koh SJ, Kim JM, Kim IK, Ko SH, Kim JS. Anti-inflammatory mechanism of metformin and its effects in intestinal inflammation and colitisassociated colon cancer. J Gastroenterol Hepatol 2014;29:502-510. doi:10.1111/jgh.12435

70. Freckelton J, Evans JA, Croagh D, Moore GT. Metformin use in diabetics with diverticular disease is associated with reduced incidence of diverticulitis. Scand J Gastroenterol 2017;52:969-972. doi:10.1080/0 0365521.2017.1325930

71. Ajala O, English P, Pinkney J. Systematic review and meta-analysis of different dietary approaches to the management of type 2 diabetes1-3. Am J Clin Nutr 2013;97:505-516. doi:10.3945/ajcn.112.042457

72. Tanaka S, Yoshimura Y, Kamada C, et al. Intakes of dietary fiber, vegetables, and fruits and incidence of cardiovascular disease in Japanese patients with type 2 diabetes. Diabetes Care 2013;36:39163922. doi: $10.2337 /$ dc13-0654

73. He M, Van Dam RM, Rimm E, Hu FB, Qi L. Whole-grain, cereal fiber, bran, and germ intake and the risks of all-cause and cardiovascular diseasespecific mortality among women with type 2 diabetes mellitus. Circulation 2010;121:2162-2168. doi:10.1161/CIRCULATIONAHA.109.907360

74. Haboubi NY, Alqudah M. Pathology and pathogenesis of diverticular disease and patterns of colonic mucosal changes overlying the diverticula. Dig Dis 2012;30:29-34. doi:10.1159/000335704

75. Lin X, Li J, Ying M, Wei F, Xie X. Diabetes increases morbidities of colonic diverticular disease and colonic diverticular hemorrhage: A systematic review and meta-analysis. Am J Ther 2017;24:e213-e221. doi:10.1097/MJT.0000000000000410 